Article

\title{
One-Step Reinforcement and Deacidification of Paper Documents: Application of Lewis Base-Chitosan Nanoparticle Coatings and Analytical Characterization
}

\author{
Zhihui Jia ${ }^{1}$, Chun Yang ${ }^{1,2}$, Fangnan Zhao ${ }^{1}$, Xiaolian Chao ${ }^{1}$, Yuhu Li ${ }^{1, *}$ and Huiping Xing ${ }^{1, *}$ \\ 1 Engineering Research Center of Historical Cultural Heritage Conservation, Ministry of Education, School of \\ Materials Science and Engineering, Shaanxi Normal University, Xi'an 710119, China; \\ jzh1988@snnu.edu.cn (Z.J.); 152353@snnu.edu.cn (C.Y.); zfn@snnu.edu.cn (F.Z.); chaoxl@snnu.edu.cn (X.C.) \\ 2 Emperor Qinshihuang's Mausoleum Site Museum, Xi'an 710600, China \\ * Correspondence: liyuhu@snnu.edu.cn (Y.L.); xhp@snnu.edu.cn (H.X.); Tel.: +86-29-8153-0715 (H.X.)
}

Received: 9 November 2020; Accepted: 11 December 2020; Published: 15 December 2020

check for updates

\begin{abstract}
To delay acidification and deterioration during natural aging, deacidification and reinforcement of paper manuscripts have been the most important technologies to prolong the life of objects. Herein, a novel approach for the conservation of paper manuscripts is proposed using chitosan nanoparticles as Lewis base that leads to both deacidification and strengthening of paper in one-step. Chitosan nanoparticles were prepared through physical ball grinding method and characterized via scanning electron microscopy (SEM), X-ray diffraction (XRD), laser particle size analyzer (LPSA), Fourier transform infrared spectroscopy (FTIR), and atomic force microscope (AFM). To evaluate the resistance of chitosan nanoparticle coating, the mechanical properties of paper after artificial aging were evaluated using dry heat and hygrothermal accelerated aging methods. The SEM, EDX, and X-ray Photoelectron Spectroscopy (XPS) were used to analyze the interaction mechanism between chitosan and Shuxuan paper. The results show that the coated paper had superior durability with respect to $\mathrm{pH}$, tensile strength, and folding endurance. There was a presence of protonated amines in the form of ammonium salts due to ionic bindings with free $\mathrm{H}^{+}$in the acidified paper, and the remaining $-\mathrm{NH}_{2}$ could be used as a base reserve. Finally, the resulting coated papers displayed good antibacterial properties.
\end{abstract}

Keywords: reinforcement; deacidification; paper document conservation; chitosan nanoparticle coating

\section{Introduction}

Besides several industrial applications, cellulose-based materials, such as paper and canvas, which are primarily composed of cellulose, provide the most common types of support for works of art. Cellulose in historical manuscripts is subjected to several decay processes that may lead to irreversible degradation and ultimate loss of valuable information [1,2]. Cellulose is a linear polymer consisting of several hundred to over ten thousand d-glucose units linked to each other by a $\beta$-(1,4)-glycosidic bond. The degree of polymerization (DP) of native cellulose can vary between 7000 and 15,000 depending on the plant species [3]. The hydrolysis of $\beta-(1,4)$-glycosidic bonds of cellulose linear chains is catalyzed by acidity and is the main cause of cellulose-based material degradation, reducing the mechanical properties of cellulose-based materials [4]. The hydrolysis reaction is affected by multiple factors such as $\mathrm{pH}$, temperature, moisture content, and degree of crystallinity, and can be described using a three-step mechanism $[5,6]$, resulting in a self-accelerating reaction $[7,8]$.

The preservation and protection of existing acidic historical paper documents have long been an important issue for libraries, archives, and museums. To delay acidification and deterioration during 
natural aging, deacidification and reinforcement have been the most important technologies to prolong the life of objects [9]. Neutralization of the acids present on the surface or interior of paper is the main chemical strategy for acidic historical paper documents. At present, the deacidification strategy based on the Acid-Base Proton Theory mainly consists of aqueous alkaline solutions of metal hydroxide or oxide nanoparticles dispersed in organic solvents. Owing to better alkaline reserve, lower toxicity, and moderate price, aqueous inorganic alkaline solution is widely accepted compared with the organic base [10]. $\mathrm{Ca}(\mathrm{OH})_{2}$ or $\mathrm{Ca}\left(\mathrm{HCO}_{3}\right)_{2}$ aqueous solutions are most commonly used due to their excellent deacidification performance than others [11-13]. However, physical changes in the appearance of paper are usually caused due to the drying process of aqueous deacidification. Although $\mathrm{MgO}$ nanoparticles dispersed in perfluorinated heptane have been successfully commercialized for paper deacidification due to their good deacidification performance and alkaline reserve [14], their employment is limited for paper conservators because of white sediments on the paper surface [15].

The reinforcement of acidic historical paper documents can greatly improve the mechanical properties of paper. In general, Japanese tissue or Chinese handmade paper is adhered to the blank side of a single-sided print to increase its mechanical strength. The polyester film encapsulation, silk lamination, and radiation polymerization are often used to improve the reinforcement of double-sided prints [16-19]. For the conservation of acidic historical paper documents, in general, two separate steps of deacidification and reinforcement are used. This method not only increases the thickness of the paper but also amplifies the workload and damage risk. Therefore, one-step deacidification and reinforcement for the conservation of paper documents is urgently required. In recent works, successful methods for deacidification and reinforcement of the acidic historical paper documents have been described. The double-sided prints can be deacidified and reinforced through lining with Japanese tissue by using wheat starch paste or carboxymethyl cellulose aqueous solution of $\mathrm{Ca}(\mathrm{OH})_{2}$ [20]. Coating of $\mathrm{ZnO}$-cellulose composites on the paper surface has good durability and antibacterial properties [21]. Calcium hydroxide nanoparticles in hydroalcoholic gelatin solutions (GeolNan) have been developed for the deacidification and strengthening of papers containing iron gall ink [22]. The Polyamidoamines were used for the conservation of irongall inked paper [23].

Chitosan (CS) is derived from chitin and is the second most abundant polysaccharide after cellulose. Owing to the characteristics of biocompatibility, non-toxicity, and biodegradability, chitosan and chitosan nanomaterials can be used in various applications [24], such as health care [25-32], antioxidant and antifungal activity [33-35], food [36], agriculture, etc. [37-40]. As the structure of chitosan is similar to cellulose (Figure 1), it has shown promising results in the surface treatment of paper to enhance barrier and mechanical properties. The present amino groups in the chitosan backbone structure can interact with $\mathrm{H}^{+}$[41,42]; however, to the best of our knowledge, there is currently no report on the use of this property for one-step deacidification and strengthening of paper. Herein, chitosan nanoparticles, such as Lewis base, were applied for one-step reinforcement and deacidification of paper documents. Chitosan nanoparticles are prepared through the ball milling method and characterized via SEM, XRD, LPSA, and FTIR. The interaction mechanism between chitosan and acid paper was analyzed using SEM, EDX, and XPS. Comparative experiments have been performed between coated paper with the chitosan nanoparticles and uncoated paper using $\mathrm{pH}$, tensile strength, and folding endurance as the parameters. To evaluate the resistance of chitosan nanoparticle coating, the degradation of mechanical properties of paper after artificial aging was evaluated via tensile strength and folding endurance tests using dry heat and wet heat accelerated aging methods. Finally, the antimicrobial activity of the chitosan-nanoparticle-coated paper was also assessed for four common fungi that are found in archives and museums. 


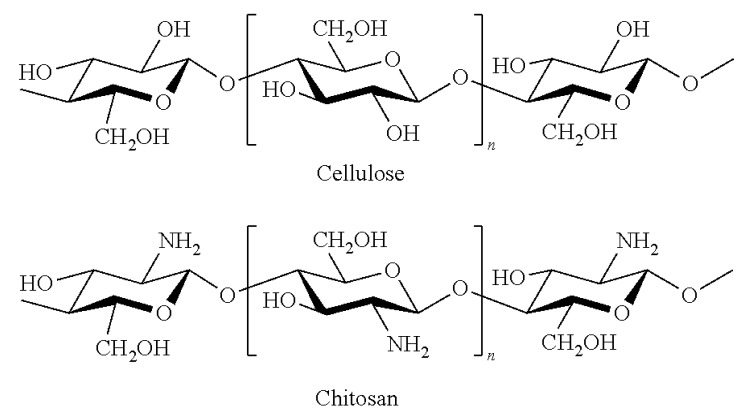

Figure 1. Structure of cellulose and chitosan.

\section{Materials and Methods}

\subsection{Materials}

Chitosan (deacetylation 90\% and MW $1 \times 10^{6} \mathrm{Da}$ ) was purchased from Zhejiang Golden-shell Pharmaceutical Co. Ltd. (Yuhuan, China), while isopropanol was purchased from J\&K Scientific Ltd. (Beijing, China) The Shuxuan paper (density $40 \mathrm{~g} / \mathrm{m}^{2}$ ) of Jingxian Purple Light Paper Industry, Anhui, China, was used as a model specimen. The $\mathrm{pH}$ of the deionized water in which the Shuxuan paper is dipped was measured according to GB/T1545-2008 [43] with METTLER-TOLEDO FE28 pH Meter (Mettler Toledo, Columbus, $\mathrm{OH}, \mathrm{USA}$ ), and the measured $\mathrm{pH}$ value was about 5.0.

\subsection{Preparation Chitosan Nanoparticles}

Chitosan powders were mechanically milled in a QM-3SP2 planetary ball mill (NanDa Instrument Plant, Nanjing, China) at 400 rpm for 120 h using zirconia balls. The chitosan powders were obtained by separating the zirconia balls.

\subsection{Conservation Procedure and Characterization Methods}

The treatments for different specimens are as follows. Different masses of chitosan nanoparticles milled $(0.2,0.4,0.6,0.8,1.0$, and $1.2 \mathrm{~g})$ were dispersed in $100 \mathrm{~mL}$ isopropanol via ultrasonic treatment for $15 \mathrm{~min}$. Then, $30 \mathrm{~mL}$ dispersive solution of chitosan with different concentrations was sprayed on a $35 \times 35 \mathrm{~cm}^{2}$ paper. The samples were placed into the BCM-1000 bio-cleaning worktable, and isopropanol was completely volatilized under low wind speed. Before testing, the samples were maintained at $(23 \pm 1){ }^{\circ} \mathrm{C}$ with the relative humidity of $(50 \pm 2) \%$ for $24 \mathrm{~h}$ according to Chinese standard GB/T 10739-2002 [44] (eqv ISO 187:1990) [45] The pH value of the specimens is increased with the amount of chitosan nanoparticles increasing. The $\mathrm{pH}$ value is increased from 5.0 to 7.11 when using $1.0 \mathrm{~g}$ chitosan nanoparticles, while the $\mathrm{pH}$ value almost maintained a constant when $1.2 \mathrm{~g}$ chitosan nanoparticles was used. Based on the above experimental results, $1.0 \mathrm{~g}$ chitosan nanoparticles dispersed in $100 \mathrm{~mL}$ isopropanol was selected, and the characterization procedure for the specimens was organized as follows.

Fourier transform infrared (FT-IR) analysis of chitosan and chitosan nanoparticles was conducted using Thermo Nicolet IS10 Spectrometer (Waltham, MA, USA) in the range between 4000 and $400 \mathrm{~cm}^{-1}$ with $\mathrm{KBr}$ pellet method. X-ray diffraction (XRD) profiles of chitosan and chitosan nanoparticles were recorded using DX-2700 X-ray diffractometer (Dandong Haoyuan Instrument Co. Ltd., Dandong, China) with $\mathrm{Cu} \mathrm{K} \alpha$ radiation. Chitosan after ball grinding was ultrasonically dispersed in the anhydrous ethanol in the concentration of $0.01 \%(v / v)$ for $20 \mathrm{~min}$. The Beckman LS13320 laser particle size analyzer (Beckman Coulter, Brea, CA, USA) was used to measure the particle size of chitosan and chitosan nanoparticles. The morphology of nanoparticles and specimens was analyzed with a scanning electron microscope (FEI, Quanta 200, Hillsboro, OR, USA). A silicon wafer was used as the base surface and tested after gold spray treatment. Analyses were performed at low vacuum (1-150 Pa) at the accelerating voltage of $5 \mathrm{kV}$ and magnification of $40 \times$. SEM images and elemental maps were generated 
using a HITACHI SU3500 SEM (Hitachi, Tokyo, Japan) coupled with an AMETEK EDAX Octane Elite EDX System at the magnification of 150x. An atomic force microscope (AFM) (Bruker, Billerica, MA, USA) was employed to observe the longitudinal direction of chitosan after ball grinding, i.e., in the direction of the height of particle. The closed-loop noise level in the XY direction was $<0.15 \mathrm{~nm}$. Open loop noise level in $X Y$ direction was $<0.10 \mathrm{~nm}$, while the $Z$ direction closed loop noise level was $<0.035 \mathrm{~nm}$. The $X Y$ scanning range was $12.5 \times 12.5 \mu \mathrm{m}^{2}$, and the vertical scanning range was $10 \mathrm{~nm}$. The polished silicon wafer was used to prepare the concentrated sulfuric acid in 7:3 ratio and mixture of $30 \%$ hydrogen peroxide solution at $90^{\circ} \mathrm{C}$ for $1 \mathrm{~h}$. Then, the sample was dropped on the silicon wafer and dried naturally for further tests. X-ray Photoelectron Spectroscopy (XPS) (Shimadu, Tokyo, Japan) was used to analyze the key elements on the Shuxuan paper surface before and after treatment with chitosan nanoparticles. $\mathrm{Al} \mathrm{K} \alpha(1486 \mathrm{eV})$ was used as the $\mathrm{X}$-ray source, wherein, the $\mathrm{X}$-ray gun was $12.5 \mathrm{kV} \times 20 \mathrm{~mA}, 250 \mathrm{~W}$, general energy was $100 \mathrm{eV}$, step length was $0.1 \mathrm{eV} / \mathrm{s}$, vacuum chamber pressure was less than $1 \times 10^{-6} \mathrm{~Pa}$, and resolution was $0.8 \mathrm{eV}$ (Ag-like $\mathrm{Al}$ target). The scanning was performed three times and normalized.

The tensile strength of $270 \times 15 \mathrm{~mm}$ size specimens was measured with QT-1136PC universal material testing machine (Gaotai Testing Instrument Co., Ltd, Dongguan, China) as per ISO 1924-2:1994 method [46]. The folding endurance of the $150 \times 15 \mathrm{~mm}^{2}$ size specimens was measured with the YT-CTM tester according to ISO 5626:1993 method [47]. The samples were cut according to the machine (MD) and cross directions (CD) of the paper.

Dry heat and hygrothermal accelerated aging methods were evaluated to evaluate the resistance of chitosan nanoparticle coating. The dry heat accelerated aging test was performed in a DHG-9245A aging chamber at $105^{\circ} \mathrm{C}$ for $72 \mathrm{~h}$ according to ISO 5630-1:1991 [48]. The hygrothermal accelerated aging test was performed in a DHG-9245A aging chamber at $80^{\circ} \mathrm{C}$ and $65 \%$ relative humidity $(R H)$ for $72 \mathrm{~h}$ as per GB/T 22894-2008 [49]. The $\mathrm{pH}$ of the deionized water in which the paper is dipped was measured according to GB/T1545-2008 with METTLER-TOLEDO FE28 pH Meter.

Aspergillus niger, Aspergillus flavus, Chaetomium globosum, Aspergillus fumigatus are the model species of fungi found in the archives or museum. To evaluate the antifungal activity of the coated paper, experiments were performed to test the durability of antifungal effect on the specimens. All the materials and vessels were sterilized in advance by autoclaving, and the tests were performed in an electroheating standing-temperature incubator in favorable conditions for fungal development (high humidity and temperature). The PDA medium (GB/T4789.15-2003) [50] and czapek's medium (GB/T4789.28-2003) [51] were used for colony breeding. Shuxuan paper samples $(\varnothing=55 \mathrm{~mm})$ per treatment were treated with the chitosan nanoparticles and placed over medium in a petridish, respectively, inoculated with the four fungal species, wherein $0.1 \mathrm{~mL}$ inoculum concentration was $1 \times 10^{8}$ spores $\mathrm{mL}^{-1}$, and incubation was performed at $22^{\circ} \mathrm{C}$. The non-inoculated samples were used as the contamination controls. The fungal growth was assessed after two weeks of incubation. This step is commonly employed by the paper conservators when dealing with paper objects affected by fungi $[52,53]$. Fungal growth was quantified by the measurement of colonized areas and the determination of biomass dry weight [54]. The \% of growth inhibition was calculated using Equation (1), where $A_{\mathrm{NT}}$ is the average colonized area on the untreated control samples and $A_{\mathrm{T}}$ is the average colonized area on the treated samples [55]:

$$
\% \text { inhibition }=\frac{A_{\mathrm{NT}}-A_{\mathrm{T}}}{A_{\mathrm{NT}}} \times 100
$$

\section{Results and Discussion}

\subsection{Characterization of Chitosan Nanoparticles}

The chitosan nanoparticles were characterized via SEM, XRD, LPSA, and FTIR. Figure 2a,b show the SEM images of the original chitosan particles and chitosan particles after ball milling for $120 \mathrm{~h}$. There is significant difference between the particle sizes. In addition, the particle size distribution of 
chitosan without ball grinding is non-uniform and its morphology is irregular, and the average particle size is larger than 5 microns. After ball grinding for $120 \mathrm{~h}$, the shape became spherical, with a diameter of less than $100 \mathrm{~nm}$.

Figure 3 displays the size distribution of chitosan after ball milling. The size of original chitosan particles is about $200 \mu \mathrm{m}$, and the size of milled chitosan particles is about $75 \mathrm{~nm}$, implying that the milling process reduced the chitosan particles size. The results of chitosan particle height after ball grinding are presented in Figure 4. The z-axis height is $10 \mathrm{~nm}$, the nanoparticles had similar thickness, and the dispersion and molecular size distribution are relatively uniform. Combined with Figures $2-4$, the results show that the chitosan powder prepared by physical ball grinding was spherical in shape, with a diameter of less than $100 \mathrm{~nm}$.
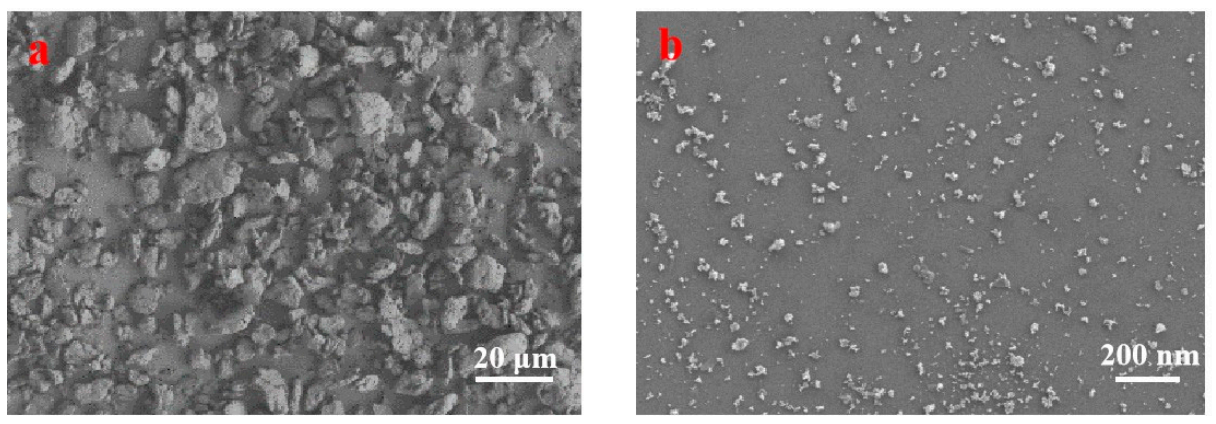

Figure 2. SEM surface images of chitosan: (a) original chitosan; (b) milled chitosan.
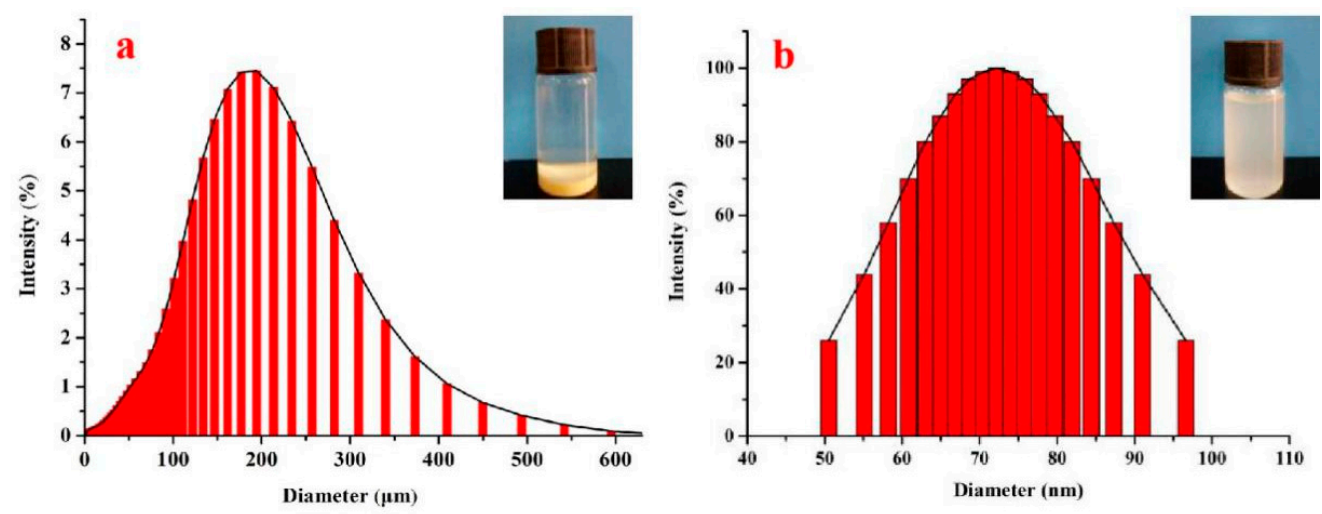

Figure 3. Particle size distribution: (a) original chitosan; (b) milled chitosan.

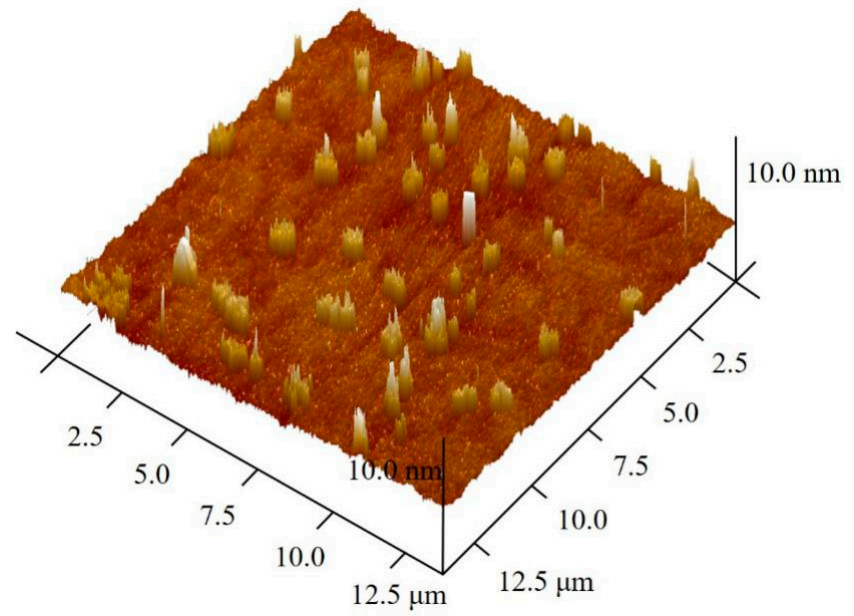

Figure 4. Atomic force microscope (AFM) images of chitosan after ball milling. 
Figure 5 presents the FTIR spectra of chitosan and chitosan nanoparticles. Absorption bands due to stretching vibration of $\mathrm{OH}$ associated with pyranose ring overlapped with that of $\mathrm{NH}_{2}$ associated with primary amines and both occurred at $3425 / 3448 \mathrm{~cm}^{-1}[56,57]$. As shown in Figure 5, the absorption peak of symmetric stretching vibration of $\mathrm{CH}$ in pyranose ring is found at $2874 \mathrm{~cm}^{-1}$. The carbonyl stretching vibration (amide-I), $\mathrm{N}-\mathrm{H}$ stretching vibration (amide-II), and $\mathrm{C}-\mathrm{N}$ stretching vibration (amide-III) of chitosan are indicated by their absorption at 1635, 1579, and $1424 \mathrm{~cm}^{-1}$, respectively [58-60]. Notably, $1164 \mathrm{~cm}^{-1}$ is the symmetric stretching vibration of $\mathrm{C}-\mathrm{O}-\mathrm{C}$ and $1073 \mathrm{~cm}^{-1}$ is the characteristic absorption peak of ether bond stretching vibration in the pyrenoid ring [49]. Comparing the infrared absorption spectra of chitosan and chitosan nanoparticles, the type and location of the characteristic absorption peak basically did not change, while the absorption peak of chitosan nanoparticles demonstrated a moving trend, indicating that the intermolecular and intramolecular hydrogen bonds of chitosan decreased. These results suggest that chemical changes of chitosan are not undergone, with respect to structure, upon reduction to nanoparticles.

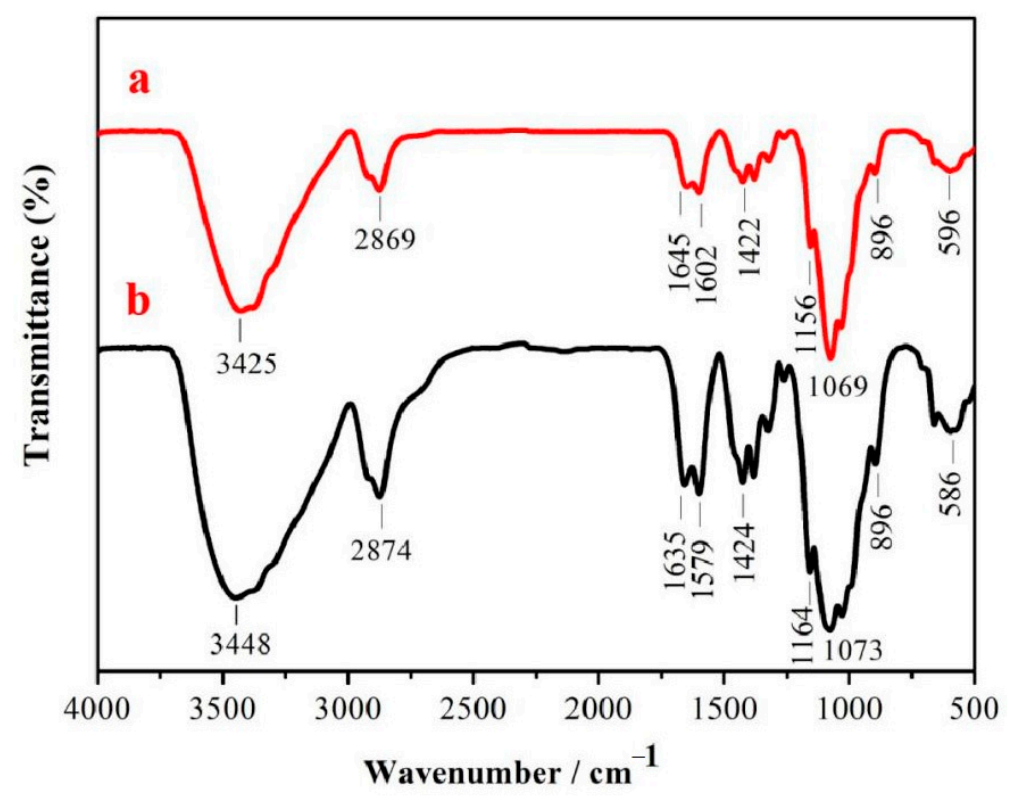

Figure 5. FTIR spectra: (a) chitosan nanoparticles; (b) chitosan.

XRD patterns of the chitosan and chitosan nanoparticles are obtained, and the corresponding results are presented in Figure 6. As shown in Figure 6, chitosan displayed a strong diffraction peak at around $20.1^{\circ}$, which is associated with the mixture of (001) and (100) planes that belong to the monoclinic system and one weak diffraction peak at $10.6^{\circ}$ [61]. XRD of chitosan nanoparticles displayed only one peak at $20.1^{\circ}$. To compare with the XRD spectrum of chitosan powder, the $10^{\circ}$ diffraction peak disappeared completely, the $20^{\circ}$ diffraction peak relative intensity decreased significantly, and the amorphous area relatively increased. This is because the process of milling disrupts the crystalline regions of chitosan and produces highly amorphous nanoparticles. 


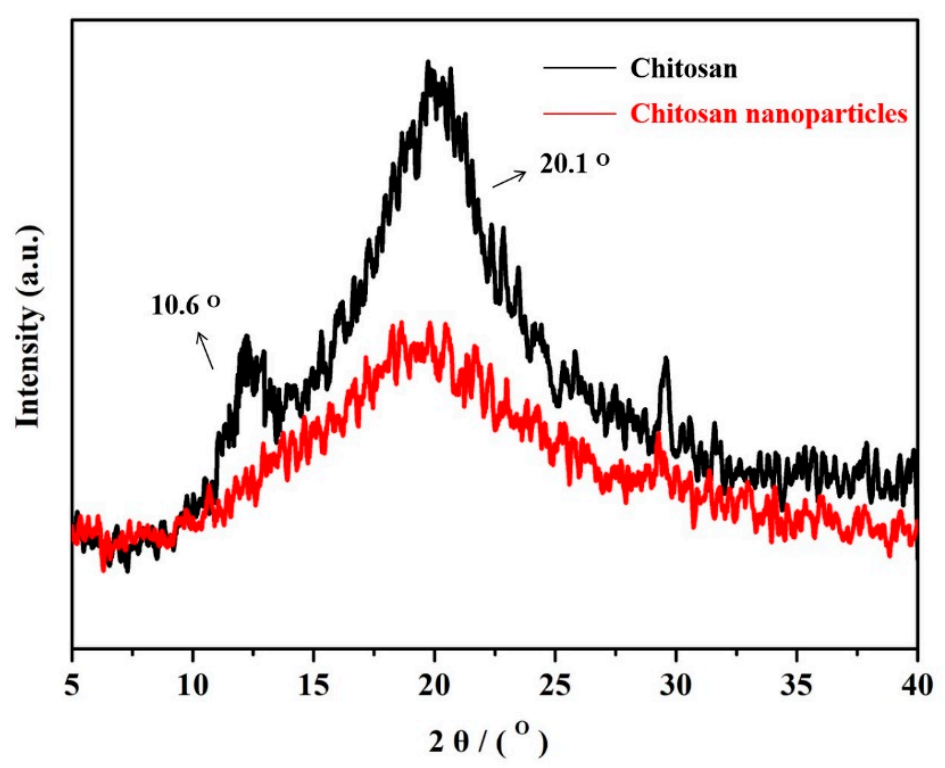

Figure 6. XRD patterns of the original chitosan and chitosan nanoparticles.

\subsection{Conservation Applications of Chitosan Nanocomposites}

The decrease in mechanical properties and acidity of the paper are prime indicators of paper deterioration [62]. Generally, the tensile strength and folding endurance are used to characterize the degradation related to the extent of oxidation and hydrolysis of cellulosic fibers of paper [63]. The comparison of tensile strength and folding endurance between the uncoated paper and the one coated with chitosan nanoparticles of paper and after accelerated aging tests is shown in Figure 7. Both the tensile strength and folding endurance are increased for the coated paper with chitosan nanoparticles as compared to the uncoated paper. It can be seen that the tensile strength and folding endurance at cross directions (CDs) of treated samples increased by $55.7 \%$ and $50.0 \%$, respectively. The folding endurance of untreated samples at cross directions (CDs) decreased by $50.0 \%$ and $83.3 \%$ after hydrothermal and dry heat ageing, while treated samples decreased by $11.1 \%$ and $22.2 \%$, respectively. The tensile strength of untreated samples at cross directions (CDs) decreased by $35.8 \%$ and $55.3 \%$ after hydrothermal and dry heat ageing, while treated samples decreased by $4.6 \%$ and $6.3 \%$, respectively. There is a significant difference between the MD and CD of the paper about tensile strength and folding endurance of the paper, and, in general, MD is greater than CD. Therefore, the Chitosan nanoparticles may play an important role in improving the mechanical properties of the paper. The coated papers have better thermal and wet stability and clearly exhibit a lower loss of paper strength than the uncoated papers. This is because the chitosan nanoparticles go deep inside the fiber, and the formed protective layer can repair the fiber defects and cracks by bridging the loose fibers and fillers. In addition, as per Figure 8 , the $\mathrm{pH}$ of the coated papers is generally increased by chitosan nanoparticles. The $\mathrm{pH}$ of paper coated with chitosan nanoparticles increased from 5.0 to 7.11, due to the presence of acid in the paper. Compared with the uncoated paper, the paper treated with chitosan nanoparticles displayed a superior durability. 

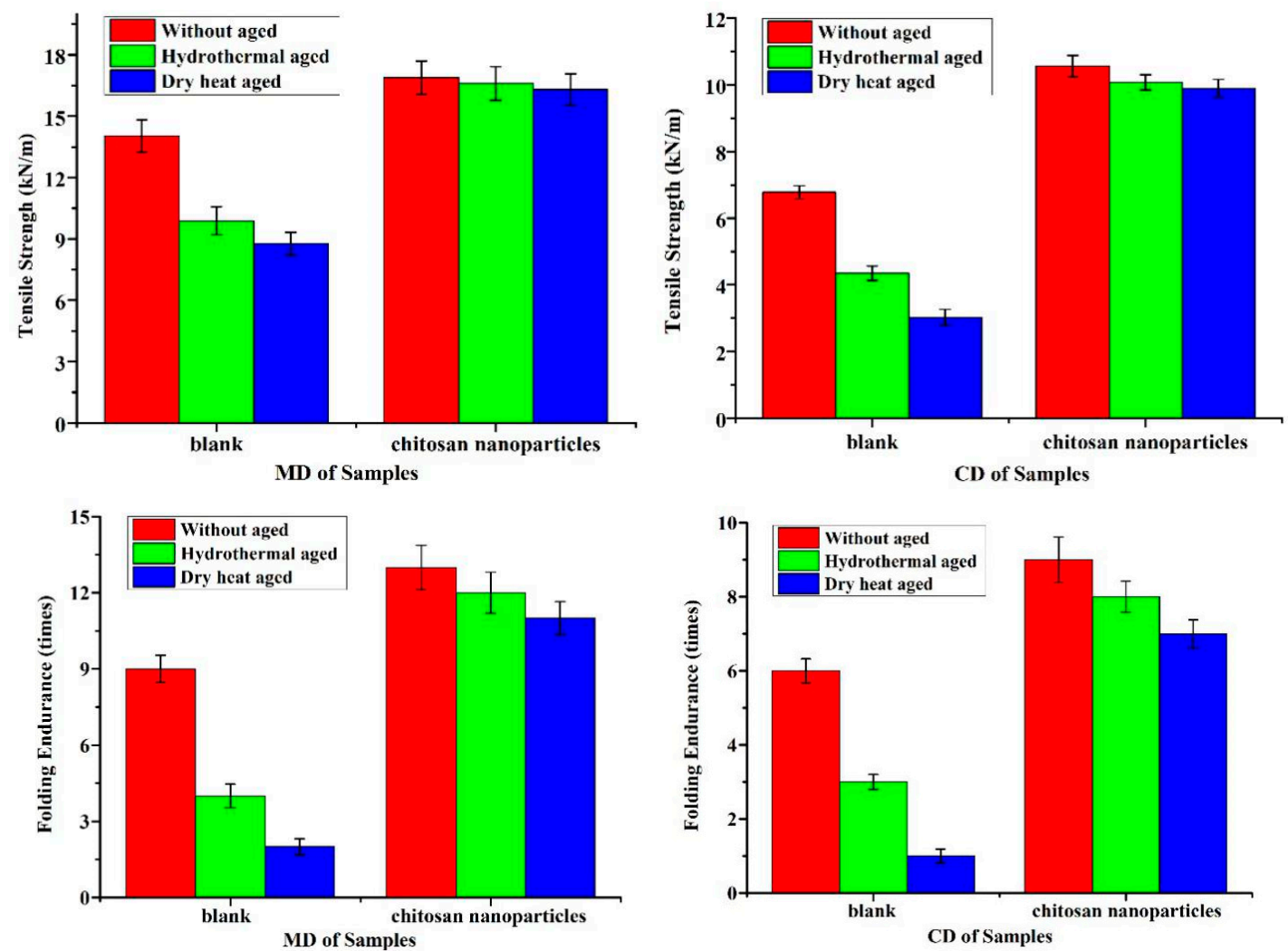

Figure 7. Tensile strength and folding endurance of uncoated and coated paper before and after accelerated aging tests.

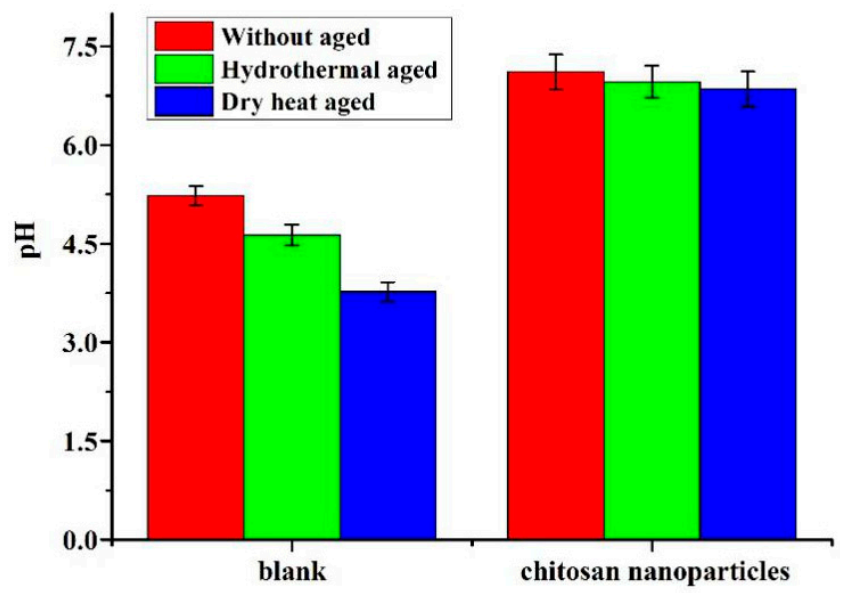

Figure 8. $\mathrm{pH}$ of uncoated and coated paper before and after accelerated aging tests.

Figure 9 presents an SEM image of the morphology of paper specimens before and after treatment with chitosan nanoparticles. As shown in Figure 9a, the cellulose fibers of the untreated paper specimens are entwined with wide pores. The chitosan nanoparticles are attached to the surface of the paper fiber and the pores in the internal fiber mesh are filled with these nanoparticles forming a protective layer (Figure $9 \mathrm{~b}$ ). The components of the nanoparticles are characterized by EDX mapping as shown in Figure 9c. Apart from $\mathrm{C}$ and $\mathrm{O}$ elements, $\mathrm{N}$ element is found in the surrounding area (Figure 9b Area 1). After hygrothermal aging, the chitosan nanoparticles demonstrated a membrane phenomenon (Figure 9d) and covered the fiber surface. After dry heat aging, the chitosan nanoparticles remained granular and dispersed on the surface of the paper and in cracks between the paper fibers (Figure 9e). Combined with Figure 7, the formed protective layer attached to the surface of the paper 
increased the mechanical strength of the paper in the process of reinforcing the paper. And the wrapped and compact fiber can prevent possible degradation due to temperature and humidity.
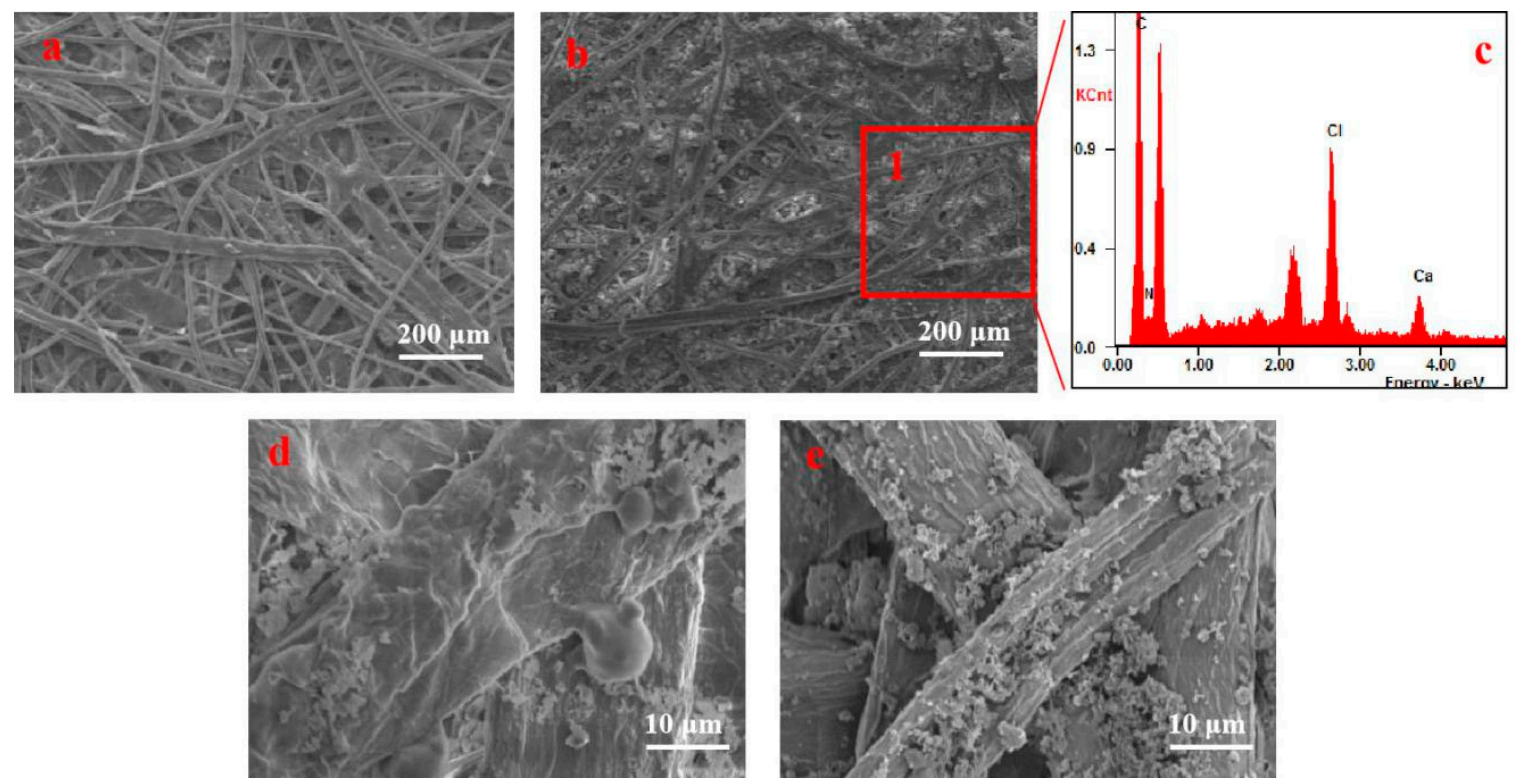

Figure 9. SEM surface images of paper: (a) before treatment with chitosan nanoparticles; (b) after treatment with chitosan nanoparticles; (c) EDX spectrum collected at Area 1; (d) treated paper after hygrothermal aging; (e) treated paper after dry heat aging.

XPS spectra of high-resolution scans of the key elements on Shuxuan paper surface before and after treatment with chitosan nanoparticles are obtained to gain further insights into the adsorption mechanism (Figure 10). Compared with the treated paper, the paper before treatment has $\mathrm{C} 1 \mathrm{~s}$ and $\mathrm{O} 1 \mathrm{~s}$ peaks. As the raw material is not processed, the paper cellulose is composed of $\mathrm{C}, \mathrm{H}$, and $\mathrm{O}$ elements. After the treatment with chitosan nanoparticles, $\mathrm{N} 1$ s peak of the chitosan nanoparticle-coated paper appeared, which indicates that the elemental composition of the paper surface is changed after the treatment. This means that $\mathrm{N}$ elements are introduced into the acidified paper through chitosan nanoparticles. Combined with Figure $9 \mathrm{~d}$, the protonation of the amino group of chitosan nanoparticles led to good solubility in water and excellent film-forming property.

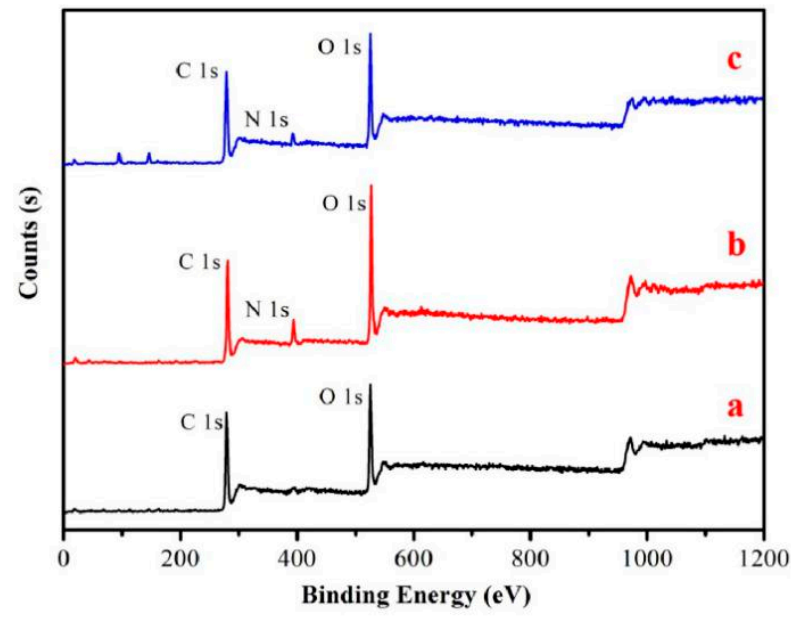

Figure 10. Wide-scan X-ray photoelectron spectroscopy (XPS) spectra of samples: (a) uncoated paper; (b) chitosan nanoparticles; (c) samples coated with chitosan nanoparticles. 
The curves of N 1s were fitted using the XPS Peak Fit software (V4.1), and the analytical spectra of $\mathrm{N} 1 s$ are presented in Figure $11 \mathrm{a}, \mathrm{b}$. The $\mathrm{N} 1 \mathrm{~s}$ of the original chitosan only had one peak after fitting (Figure 11a). The binding energy at $399.7 \mathrm{eV}$ corresponds to $-\mathrm{NH}_{2}$, which reflects the original molecular structure of chitosan. There are two peaks in Figure $11 \mathrm{~b}$. The peak at $399.7 \mathrm{eV}$ is assigned to $\mathrm{NH}_{2}$ chemical bonding, while the peak at $401.4 \mathrm{eV}$ is assigned to amino groups in the ammonium form $\left(\mathrm{NH}_{3}{ }^{+}\right.$) [64]. Considering the $\mathrm{pKa}$ of chitosan amine groups (ca. 6.5), both forms, $\mathrm{NH}_{2}$ and $\mathrm{NH}_{3}{ }^{+}$, are likely to be present in the chitosan membranes [65]. The upward shift of the electron binding energy to $401.4 \mathrm{~V}$ for the peak assigned to $\mathrm{NH}_{3}{ }^{+}$groups suggests the presence of protonated amines in the form of ammonium salts. This is because of the ionic bonding with free $\mathrm{H}^{+}$in the acidified paper. The remaining $-\mathrm{NH}_{2}$ can be used as a base reserve. As shown in Figure 7, the increase in strength of the paper treated with chitosan nanoparticles is due to the formation of chitosan protective layer when the nanoparticles combined with $\mathrm{H}^{+}$. The possible interaction mechanism is shown in a schematic in Figure 12. The isopropanol suspension of chitosan nanoparticles is sprayed on acidic paper and used as Lewis base to combine with free acids in the paper forming ammonium salt. Under the action of water molecules, the film is formed, and there is molecular interaction between chitosan and cellulose. Finally, the tensile strength of the paper increased by 1.557 times, and the folding resistance increased by 1.5 times compared to the untreated samples.
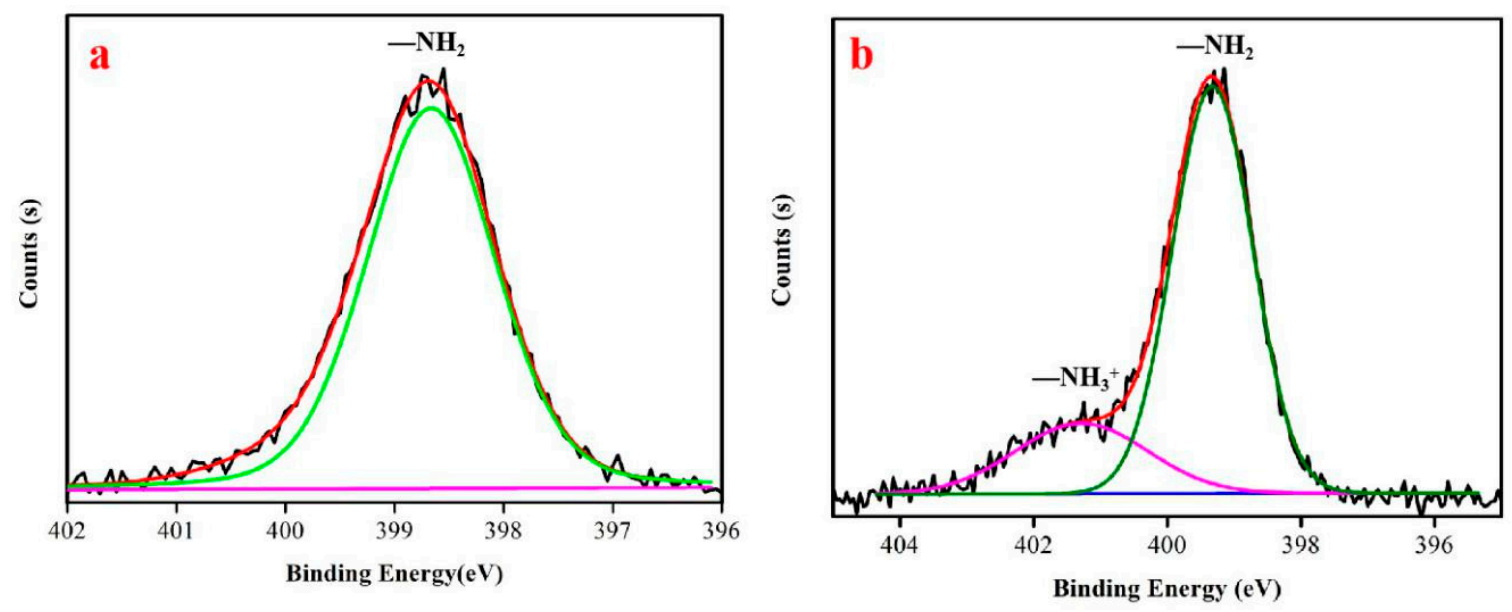

Figure 11. N 1s peaks in analytical spectra: (a) chitosan nanoparticles; (b) Shuxuan paper coated with chitosan nanoparticles.

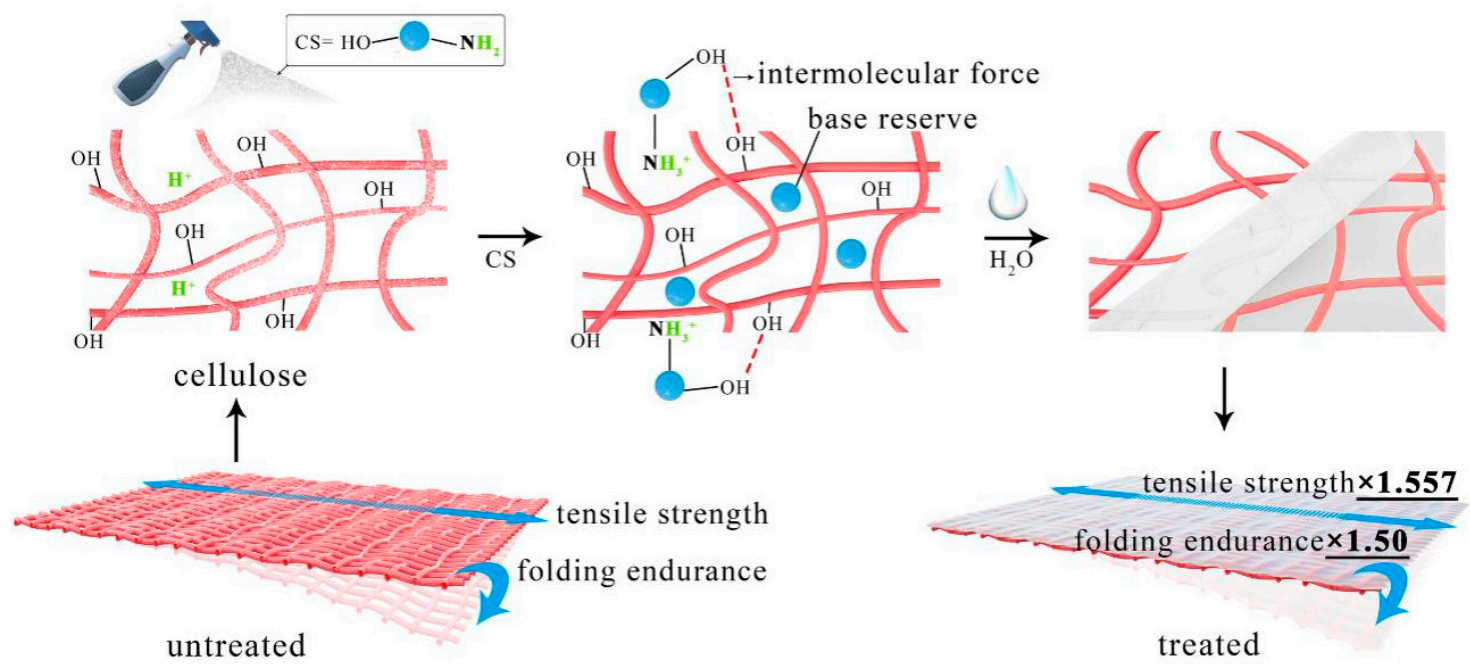

Figure 12. Schematic of possible interactions between CS nanoparticles, cellulose, and free acid. 
The durability of the antifungal effect was, respectively, evaluated for the different fungal inoculum for treated and untreated paper samples at two weeks. As presented in Figure 13, there was greater fungal growth on the untreated paper samples. Untreated paper vaccinated for aspergillus Niger in a petri dish displayed clear stellate distribution. Aspergillus flavus presented a yellow powder on the surface of the untreated circular paper. Aspergillus fumigatus mycelium was distributed similar to "smoke" on the surface of the untreated circular paper. Due to the fast growth of mycelia, the mold colonies easily spread and expanded, further masking the effective information. In addition, some fungal metabolites can reduce the paper strength and pose a threat to consumer health. The paper samples that were treated with chitosan nanoparticles displayed a significant preventive antifungal effect against the fungal inoculum. Under the same conditions, the mold did not grow on the paper and the culture plate had slightly more prominent. Aspergillus fumigatus and the fungal growth inhibition was about $92 \%$ (Table 1). However, the inhibition effect was significantly better compared with the untreated samples. This is because when the chitosan nanoparticles are protonized under acidic conditions, electrostatic interactions between the positive charges from chitosan chains and negatively charged phospholipids affect the cell membranes. In addition, the protonized nanoparticles become more soluble in aqueous solutions, and for this reason a better interaction with all kinds of molecules are expected to be more facilitated [66]. This phenomenon prevents the spread of mold and inhibits the growth and reproduction of mold.

Table 1. Calculated fungal growth inhibition (\%) at two weeks of incubation on the coated and uncoated paper samples. Each value is an average of three replicates $\pm S D$.

\begin{tabular}{ccccc}
\hline Samples & Aspergillus niger & Aspergillus flavus & Aspergillus fumigatus & Chaetomium globosum \\
\hline Uncoated paper samples & $4 \pm 2$ & $52 \pm 7$ & $35 \pm 4$ & $26 \pm 3$ \\
Coated paper samples & $100 \pm 0$ & $100 \pm 0$ & $92 \pm 0$ & $100 \pm 0$ \\
\hline
\end{tabular}

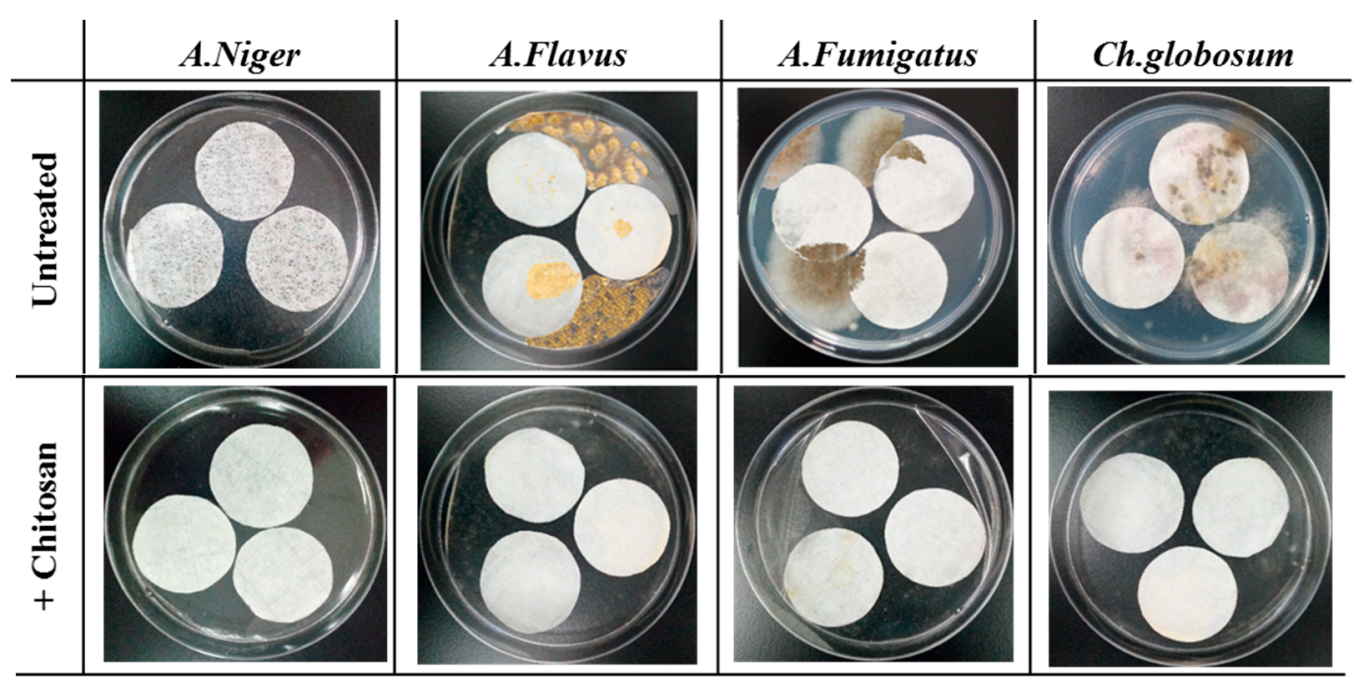

Figure 13. Images of paper samples at two weeks of incubation after preventive treatment.

\section{Conclusions}

Chitosan nanoparticles are employed for the one-step reinforcement and deacidification of paper documents. Chitosan nanoparticles are prepared via the physical ball grinding method and characterized by SEM, XRD, LPSA, and FTIR. The results show that the chitosan powder prepared by physical ball grinding is spherical in shape, with a diameter of less than $100 \mathrm{~nm}$, and the chemical changes of chitosan are not undergone, with respect to structure, upon reduction to nanoparticles. The SEM, EDX, and XPS are used to analyze the interaction mechanism between the chitosan nanoparticles and Shuxuan paper. The increase in the strength of the paper treated with chitosan nanoparticles is due to the formation of chitosan protective layer when the nanoparticles combined with $\mathrm{H}_{2} \mathrm{O}$ and $\mathrm{H}^{+}$. The $\mathrm{pH}$ 
of coated papers is generally increased from 5.0 to 7.11 and tensile strength and folding endurance are both increased for the paper coated with chitosan nanoparticles compared to the uncoated paper. To evaluate the resistance of chitosan nanoparticle coating, the degradation of mechanical properties of paper after artificial aging is evaluated by the tensile strength and folding endurance tests using dry heat and hygrothermal accelerated aging methods. The paper treated with chitosan nanoparticles displayed superior durability. The SEM, EDX, and XPS are used to analyze the interaction mechanism between the chitosan and Shuxuan paper. There is a presence of protonated amines in the form of ammonium salts due to ionic bonding with free $\mathrm{H}^{+}$in the acidified paper, and the remaining $-\mathrm{NH}_{2}$ can be used as a base reserve. The antimicrobial activity of the chitosan-nanoparticle-coated paper is also assessed with four common fungi that are found in archives and museums, and the results show that the chitosan nanoparticles have strong antibacterial properties. The as-proposed technique can provide technical support to improve the mechanical properties of acidified fragile paper. In the future, it is possible to effectively save on manpower and material resources and prevent damage to paper in the repair process.

Author Contributions: H.X. and Y.L. designed the study; Z.J., C.Y., and F.Z. performed the measurements processed experimental data; Z.J., X.C., and H.X. wrote most of the initial versions of the text. All authors contributed to research strategy, the discussion and interpretation of the results and to the final form of the text and figures. All authors have read and agreed to the published version of the manuscript.

Funding: The research is financially supported by National Natural Science Foundation of China (Grant No. 22002080), Fundamental Research Funds for the Central Universities (Grant No. GK 201903049 and 201903054), Key Research and Development Program of Shaanxi Province, China (Grant No. 2020SF-358), and Science and Technology Project of Xi'an, China (Grant No. 2020KJRC0014).

Acknowledgments: Thanks to Yuhu Li for setting up the framework of the entire project. Thanks for the fund support.

Conflicts of Interest: The authors declare no conflict of interest.

\section{References}

1. Foladi, S.; Hedayati, M.; Shokohi, T.; Mayahi, S. Study on fungi in archives of offices, with a particular focus on stachybotrys chartarum. J. Mycol. Med. 2013, 23, 242-246. [CrossRef] [PubMed]

2. Havlínová, B.; Katuččák, S.; Petrovičová, M.; Maková, A.; Brezová, V. A study of mechanical properties of papers exposed to various methods of accelerated ageing. Part I. The effect of heat and humidity on original wood-pulp papers. J. Cult. Herit. 2009, 10, 222-231. [CrossRef]

3. Fengel, D.; Wegener, G. Wood: Chemistry, Ultrastructure, Reactions; Walter De Gruyter.: Berlin, Germany; New York, NY, USA, 1984.

4. Orr, R.S.; Weiss, L.C.; Humphreys, G.C.; Mares, T.; Grant, J.N. Degradation of cotton fibers and yarns by heat and moisture. Text. Res. J. 1954, 24, 399-406. [CrossRef]

5. Harris, J.F. Acid hydrolysis and dehydration reactions for utilizing plant carbohydrates. Appl. Polym. Symp. $1975,28,131-144$.

6. Banait, N.S.; Jencks, W.P. Reactions of anionic nucleophiles with alpha-dglucopyranosyl fluoride in aqueous solution through a concerted, ANDN (SN2) mechanism. J. Am. Chem. Soc. 1991, 113, 7951-7958. [CrossRef]

7. Zhang, Y.; Bommuswamy, J.; Sinnott, M.L. Kinetic isotope effect study of transition states for the hydrolyses of alpha- and beta-glucopyranosyl fluorides. J. Am. Chem. Soc. 1994, 116, 7557-7563. [CrossRef]

8. Lundgaard, L.E.; Hansen, W.; Linhjell, D.; Painter, T.J. Aging of oil-impregnated paper in power transformers. Power Deliv. IEEE Trans. 2004, 19, 230-239. [CrossRef]

9. Zervos, S.; Alexopoulou, I. Paper conservation methods: A literature review. Cellulose 2015, 22, 2859-2897. [CrossRef]

10. Baty, J.W.; Maitland, C.L.; Minter, W.; Hubbe, M.A.; Jordan-Mowery, S.K. Deacidification for the conservation and preservation of paper-based works: A review. Bioresources 2010, 5, 1955-2023. [CrossRef]

11. Bansa, H. Aqueous deacidification-with calcium or with magnesium? Restaurator 1998, 19, 1-40. [CrossRef] 
12. Baty, J.W.; Sinnott, M.L. The kinetics of the spontaneous, proton-and $\mathrm{Al}^{\mathrm{III}}$-catalysed hydrolysis of 1,5-anhydrocellobiitol models for cellulose depolymerization in paper aging and alkaline pulping, and a benchmark for cellulase efficiency. Can. J. Chem. 2005, 83, 1516-1524. [CrossRef]

13. Botti, L.; Mantovani, O.; Orrù, M.A.; Ruggiero, D. The effect of sodium and calcium ions in the deacidification of paper: A chemo-physical study using thermal analysis. Restaurator 2006, 27, 9-23. [CrossRef]

14. Giorgi, R.; Dei, L.; Ceccato, M.; Schettino, C.; Baglioni, P. Nanotechnologies for conservation of cultural heritage: Paper and canvas deacidification. Langmuir 2002, 21, 8198-8203. [CrossRef]

15. Banik, G. Mass deacidification technology in Germany and its quality control. Restaurator 2005, 26, 63-75. [CrossRef]

16. Cernic Letnar, M.; Vodopivec, J. Protection and conservation of materials on paper. Evaluation of permanence and durability of the laminated material on paper. Restaurator 1997, 18, 177-190.

17. D'Orazio, L.; Gentile, G.; Mancarella, C.; Martuscelli, E.; Massa, V. Water-dispersed polymers for the conservation and restoration of cultural heritage: A molecular, thermal, structural and mechanical characterization. Polym. Test. 2001, 20, 227-240. [CrossRef]

18. Jiang, F.; Yang, Y.; Weng, J.; Zhang, X. Layer-by-layer self-assembly for reinforcement of aged papers. Ind. Eng. Chem. Res. 2016, 55, 10544-10554. [CrossRef]

19. Li, Q.; Xi, S.; Zhang, X. Conservation of paper relics by electrospun PVDF fiber membranes. J. Cult. Herit. 2014, 15, 359-364. [CrossRef]

20. Zhang, M.F.; Jiang, F.Z. One-step lining and deacidification of aged newspapers with double-sided writing. Restaurator 2017, 18, 1-18. [CrossRef]

21. Jia, M.H.; Zhang, X.G.; Weng, J.J.; Zhang, J.; Zhang, M.F. Protective coating of paper works: ZnO/cellulose nanocrystal composites and analytical characterization. J. Cult. Herit. 2019, 38, 64-74. [CrossRef]

22. Poggi, G.; Sistach, M.C.; Marin, E.; Garcia, J.F.; Giorgi, R.; Baglioni, P. Calcium hydroxide nanoparticles in hydroalcoholic gelatin solutions (GeolNan) for the deacidification and strengthening of papers containing iron gall ink. J. Cult. Herit. 2016, 18, 250-257. [CrossRef]

23. Isca, C.; Maggio, R.D.; Collado, N.P.; Predieri, G.; Lottici, P.P. The use of polyamidoamines for the conservation of iron gall inked paper. Cellulose 2019, 26, 1277-1296. [CrossRef]

24. Dutta, P.K.; Dutta, J.; Tripathi, V.S. Chitin and chitosan: Chemistry, properties and applications. J. Sci. Ind. Res. India 2004, 63, 20-23.

25. Jayakumar, R.; Menon, D.; Manzoor, K.; Nair, S.V.; Tamura, H. Biomedical applications of chitin and chitosan based nanomaterials: A short review. Carbohydr. Polym. 2010, 82, 227-232. [CrossRef]

26. Jayakumar, R.; Prabaharan, M.; Nair, S.; Tamura, H. Novel chitin and chitosan nanofibers in biomedical applications. Biotechnol. Adv. 2010, 28, 142-150. [CrossRef] [PubMed]

27. Ghadi, A.; Mahjoub, S.; Tabandeh, F.; Talebnia, F. Synthesis and optimization of chitosan nanoparticles: Potential applications in nanomedicine and biomedical engineering. Casp. J. Int. Med. 2014, 5, 156-161.

28. Ghadi, A.; Tabandeh, F.; Mahjoub, S.; Mohsenifar, A.; Roshan, F.T.; Alavije, R.S. Fabrication and characterization of core-shell magnetic chitosan nanoparticles as a novel carrier for immobilization of Burkholderia cepacia lipase. J. Oleo. Sci. 2015, 64, 423-430. [CrossRef]

29. Malmiri, H.J.; Jahanian, M.A.G.; Berenjian, A. Potential applications of chitosan nanoparticles as novel support in enzyme immobilization. Am. J. Biochem. Biotechnol. 2012, 8, 203-219.

30. Lopez-Leon, T.; Carvalho, E.L.S.; Seijo, B.; Ortega-Vinuesa, J.L.; Bastos-Gonzailez, D. Physicochemical characterization of chitosan nanoparticles: Electrokinetic and stability behavior. J. Colloid Interface Sci. 2005, 283, 344-351. [CrossRef]

31. Perera, U.; Rajapakse, N. Chitosan nanoparticles: Preparation, characterization, and applications. In Seafood Processing by-Products: Trends and Applications; Kim, S.K., Ed.; Springer: New York, NY, USA, 2013; pp. 371-387.

32. Shi, L.E.; Tang, Z.X.; Yi, Y.; Chen, J.S.; Xiong, W.Y.; Ying, G.Q. Immobilization of nuclease p1 on chitosanmicro-spheres. Chem. Biochem. Eng. Q. 2011, 25, 83-88.

33. Rajalakshmi, A.; Krithiga, N.; Jayachitra, A. Antioxidant activity of the chitosan extracted from shrimp exoskeleton. Middle East. J. Sci. Res. 2013, 16, 1446-1451.

34. Lin, S.B.; Chen, S.H.; Peng, K.C. Preparation of antibacterial chito-oligosaccharide by altering the degree of deacetylation of $\beta$-chitosan in a Trichoderma harzianum chitinase-hydrolysing process. J. Sci. Food Agric. 2009, 89, 238-244. [CrossRef] 
35. Huang, Y.C.; Li, R.Y. Preparation and characterization of antioxidant nanoparticles composed of chitosan and fucoidan for antibiotics delivery. Mar. Drugs 2014, 12, 4379-4398. [CrossRef] [PubMed]

36. Kardas, I.; Struszczyk, M.H.; Kucharska, M.; Van den Broek, L.A.M.; Van Dam, J.E.G.; Ciechanska, D. Chitin and chitosan as functional biopolymers for industrial applications. In The European Polysaccharide Network of Excellence (EPNOE); Research Initiatives and Results; Navard, P., Ed.; Springer-Verlag Wien: Vienna, Austria, 2012; pp. 329-375.

37. Kashyap, P.L.; Xiang, X.; Heiden, P. Chitosan nanoparticle based delivery systems for sustainable agriculture. Int. J. Biol. Macromol. 2015, 77, 36-51. [CrossRef]

38. Van, T.N.; Hanh, T.T. Application of chitosan solutions for rice production in vietnam. Afr. J. Biotechnol. 2013, 12, 382-384.

39. Wang, M.; Chen, Y.; Zhang, R.; Wang, W.; Zhao, X.; Du, Y.; Yin, H. Effects of chitosan oligosaccharides on the yield components and production quality of different wheat cultivars (Triticum aestivum L.) in northwest china. Field. Crop. Res. 2015, 172, 11-20. [CrossRef]

40. Saavedra, G.M.; Figueroa, N.E.; Poblete, L.A.; Cherian, S.; Figueroa, C.R. Effects of preharvest applications of methyl jasmonate and chitosan on postharvest decay, quality and chemical attributes of fragaria chiloensis fruit. Food. Chem. 2016, 190, 448-453. [CrossRef]

41. Bhardwaj, S.; Bhardwaj, N.K.; Negi, Y.S. Cleaner approach for improving the papermaking from agro and hardwood blended pulps using biopolymers. J. Clean. Prod. 2019, 213, 134-142. [CrossRef]

42. Rinaudo, M. Chitin and chitosan: Properties and applications. Prog. Polym. Sci. 2006, 31, 603-632. [CrossRef]

43. Paper Board and Pulp-Determination of Acidity or Alkalinity; GB/T 1545-2008; Chinese Standard: Beijing, China, 2008.

44. Paper, Board and Pulps-Standard Atmosphere for Conditioning and Testing; GB/T 10739-2002; Chinese Standard: Beijing, China, 2002.

45. Paper, Board and Pulps-Standard Atmosphere for Conditioning and Testing and Procedure for Monitoring the Atmosphere and Conditioning of Samples; ISO 187:1990; ISO: Geneva, Switzerland, 1990.

46. Paper and Board-Determination of Tensile Properties-Part 2: Constant Rate of Elongation Method (20 mm/min); ISO 1924-2:1994; ISO: Geneva, Switzerland, 1994.

47. Paper-Determination of Folding Endurance; ISO 5626:1993; ISO: Geneva, Switzerland, 1993.

48. Paper and Board-Accelerated Ageing —Part 1: Dry Heat Treatment at 105 Degrees C; ISO 5630-1:1991; ISO: Geneva, Switzerland, 1991.

49. Paper and Board-Accelerated Ageing-Moist Heat Treatment at $80^{\circ} \mathrm{C}$ and $65 \%$ Relative Humidity; GB/T 22894-2008; Chinese Standard: Beijing, China, 2008.

50. Microbiological Examination of food Hygiene-Enumeration of Molds and Yeasts; GB/T 4789.15-2003; Chinese Standard: Beijing, China, 2003.

51. Microbiolgical Examination of Food Hygiene-Stainning Methods, Culture Mediums and Reagents; GB/T 4789.28-2003; Chinese Standard: Beijing, China, 2003.

52. Florian, M. Fungal Facts—Solving Fungal Problems in Heritage Collections; Archetype Publications: London, UK, 2002.

53. Sequeira, S.O.; Cabrita, E.J.; Macedo, M.F. Fungal biodeterioration of paper: How are paper and book conservators dealing with it? An international survey. Restaurator 2014, 35, 181-199. [CrossRef]

54. Sequeira, S.O.; Phillips, A.J.L.; Cabrita, E.J.; Macedo, M.F. Ethanol as an antifungal treatment for paper: Short-term and long-term effects. Stud. Conserv. 2017, 62, 33-42. [CrossRef]

55. Ansari, N.A.; Khan, M.W.; Muheet, A. Evaluation of some fungicides for seed treatment and foliar application in management of damping-off of seedlings and blight of rapeseed caused by Alternaria brassicae. Mycopathologia 1990, 110, 163-167. [CrossRef]

56. Amiri, E.; Rahmaninia, M.; Khosravani, A. Effect of chitosan molecular weight on the performance of chitosan silica nanoparticle system in recycled pulp. BioResources 2019, 14, 7687-7701.

57. Sabazoodkhiz, R.; Rahmaninia, M.; Ramezani, O. Interaction of chitosan biopolymer with silica nanoparticles as a novel retention/drainage and reinforcement aid in recycled cellulosic fibers. Cellulose 2017, 24, 3433-3444. [CrossRef]

58. Kaya, M.; Seyyar, O.; Baran, T.; Turkes, T. Bat guano as new and attractive chitin and chitosan source. Front. Zool. 2014, 11, 1-10. [CrossRef]

59. Gbenebor, O.P.; Akpan, E.I.; Adeosun, S.O. Thermal, structural and acetylation behavior of snail and periwinkle shells chitin. Prog. Biomater. 2017, 6, 97-111. [CrossRef] 
60. Zemljic, L.F.; Valh, J.V.; Kreze, T. Preparation of antimicrobial paper sheets using chitosan. Cellul. Chem. Technol. 2017, 51, 75-81.

61. Muley, A.B.; Ladole, M.R.; Suprasanna, P.; Dalvi, S.G. Intensification in biological properties of chitosan after $\gamma$-irradiation. Int. J. Biol. Macromol. 2019, 131, 435-444. [CrossRef]

62. Conte, A.M.; Pulci, O.; Sole, R.D.; Knapik, A.; Bagniuk, J.; Lojewska, J.; Teodonio, L.; Missori, M. Experimental and theoretical study of the yellowing of ancient paper. Surf. Sci. Nanotechnol. 2012, 10,569-574. [CrossRef]

63. Kato, K.L.; Cameron, R.E. A review of the relationship between thermally accelerated ageing of paper and hornifification. Cellulose 1999, 6, 23-40. [CrossRef]

64. Amaral, I.F.; Granja, P.L.; Barbosa, M.A. Chemical modification of chitosan by phosphorylation: An XPS, FT-IR and SEM study. J. Biomater. Sci. Polymer Ed. 2005, 16, 1575-1593. [CrossRef] [PubMed]

65. Domard, A. Advances in Chitin Science. In Proceedings of the 7th International Conference on Chitin Chitosan and Euchis'97, Lyon, France, 3-5 September 1997; Jacques Andre: Lyon, France, 1997; Volume 410.

66. Matica, A.; Menghiu, G.; Ostafe, V. Antifungal Properties of Chitosans. New Front. Chem. 2017, 26, 55-63.

Publisher's Note: MDPI stays neutral with regard to jurisdictional claims in published maps and institutional affiliations.

(C) 2020 by the authors. Licensee MDPI, Basel, Switzerland. This article is an open access article distributed under the terms and conditions of the Creative Commons Attribution (CC BY) license (http://creativecommons.org/licenses/by/4.0/). 